\title{
Impairment of Early Insulin Response after Glucose Load, Rather than Insulin Resistance, is Responsible for Postprandial Hyperglycemia Seen in Obese Type 2 Diabetes: Assessment Using Nateglinide, a New Insulin Secretagogue
}

\author{
Hiroshi UCHINO, MASATAKa NIWA, TOMOAKI SHIMIZU, KyoKo NISHIYAMA aNd RYUZo KAWAMORI
}

Department of Medicine, Metabolism \& Endocrinology, Juntendo University School of Medicine, 1-1, Hongo 2-chome, Bunkyo-ku, Tokyo 113-8421, Japan

\begin{abstract}
The insulin secretory pattern as a phenotype of type 2 diabetes is an impairment in the rapid, pulsatile secretion of insulin in response to a rise in blood glucose after meal-intake. The restoration of endogenous rapid insulin secretion after oral glucose load was established for the first time by using nateglinide, which is a newly developed insulin secretagogue, in obese patients with type 2 diabetes mellitus. It was clearly demonstrated that with nateglinide, serum insulin levels were quickly raised, and glycemic response curves were almost normalized with the same amount of insulin secretion during $180 \mathrm{~min}$. Therefore, the lack of rapid, pulsatile secretion of insulin in response to glycemic rise after oral glucose load, rather than insulin resistance, is responsible for postprandial glycemic response in obese type 2 diabetes patients.
\end{abstract}

Key words: Type 2 diabetes, Early prandial insulin secretion, Nateglinide, Insulin resistance, Obesity

(Endocrine Journal 47: 639-641, 2000)

THE insulin secretory pattern as a phenotype of type 2 diabetes is of no doubt an impairment in the rapid, pulsatile secretion of insulin in response to a rise in blood glucose. Obese subjects with type 2 diabetes, especially soon after the onset of diabetes, usually exhibit postprandial hyperglycemia with delayed hyperinsulinemia. It is recognized that insulin resistance causes postprandial hyperglycemia [1, 2]. However, it is also possible that impairment of early insulin secretion in response to oral glucose load is the reason why postprandial hyperglycemia happens [3]. But so far there is no modality to stimulate en-

Received: May 29, 2000

Accepted: August 7, 2000

Correspondence to: Ryuzo KAWAMORI M.D., Ph.D., Department of Medicine, Metabolism \& Endocrinology, Juntendo University School of Medicine, 2-1-1,Hongo, Bunkyo, Tokyo 113-8421, Japan dogenous insulin secretion quickly after oral glucose load in subjects with type 2 diabetes. Nateglinide, a phenylalanine derivative, is a new non-sulfonylurea hypoglycemic agent that produces quick insulin secretion. The feature of this agent is that the stimulating effect of insulin secretion starts more quickly and disappears more rapidly than the sulfonylureas currently in use [4]. Therefore, we examined whether the impairment of early insulin response to orally administered glucose is resolved with nateglinide in obese patients with type 2 diabetes.

\section{Subjects \& Methods}

Under informed consent ten newly diagnosed obese type 2 diabetes ( 5 male and 5 female, mean age $49.3 \pm 15.6$ years (Mean $\pm S D$ ), body mass index 
(BMI) $(28.6 \pm 3.3)$ were given $75 \mathrm{~g}$ oral glucose tolerance test ( $75 \mathrm{~g}$ OGTT) twice within 3 days. At the 2nd $75 \mathrm{~g}$ OGTT, they were given $120 \mathrm{mg}$ of nateglinide 5 minutes before oral glucose load for the purpose of restoring impaired early insulin secretion. The mean homeostasis model insulin resistance index (HOMA-R) was $3.33 \pm 1.97$. Blood samples (for the determinations of serum glucose and immunoreactive insulin (IRI) concentrations) were taken before glucose load, $15 \mathrm{~min}$ after, $30 \mathrm{~min}$ after and every $30 \mathrm{~min}$ thereafter until $180 \mathrm{~min}$. The data are expressed as mean \pm SD. Paired t-test was used for comparisons between 1st and 2nd OGTT. P values $<0.05$ were considered to be statistically significant.

\section{Results}

At $60 \mathrm{~min}$ after glucose load, the blood glucose levels were decreased significantly on nateglinide, and the average area under the curve of blood glucose was decreased significantly (control $38.2 \pm 9.1 \mathrm{mmol} \cdot \mathrm{hr} / 1$
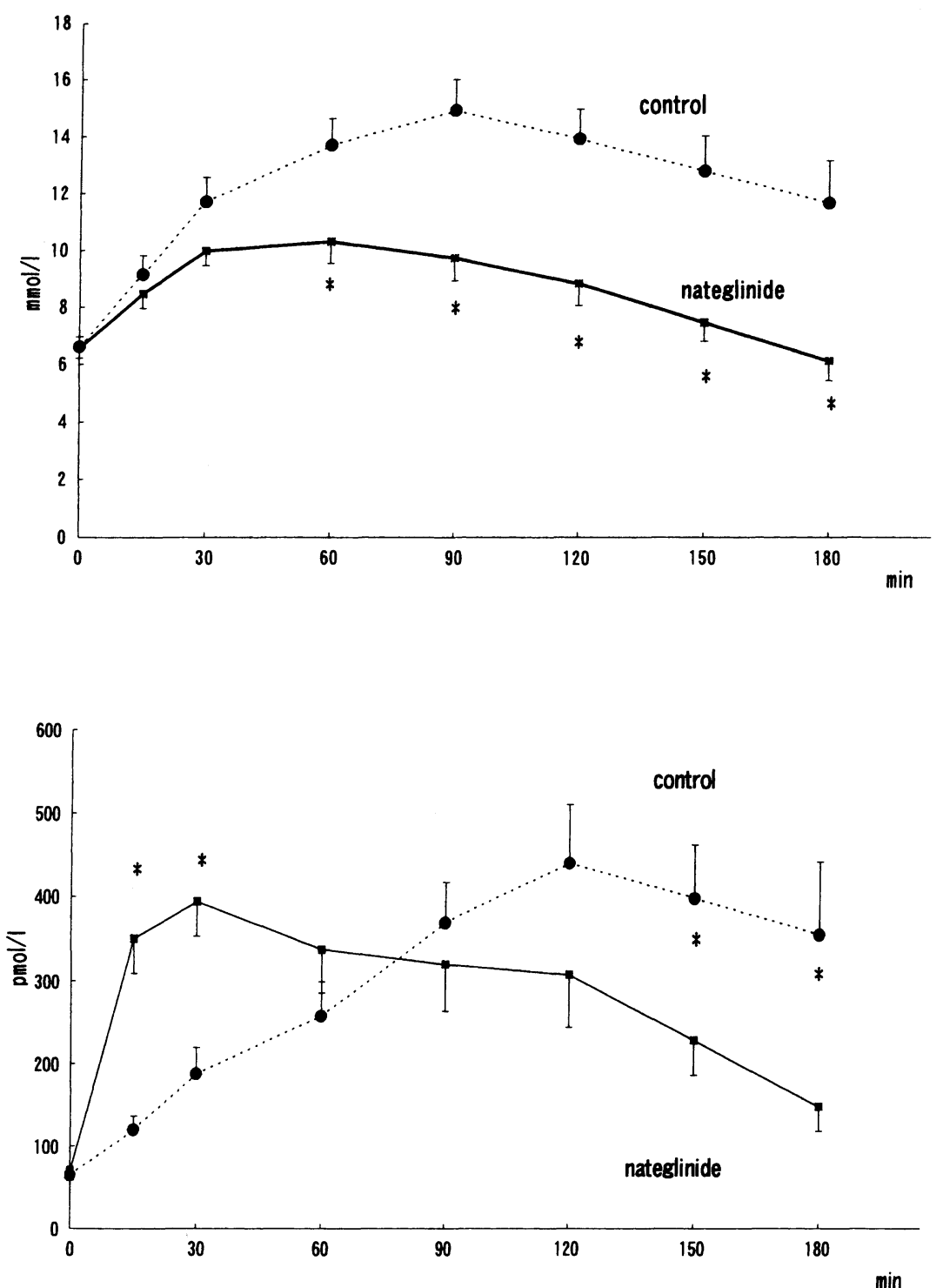

Fig. 1. Glycemic responses (upper panel) and serum insulin concentrations (lower panel) during OGTT with or without nateglinide in obese type 2 diabetes patients. Asterisks denote the significant difference as compared with those without nateglinide administration. Data are shown as mean \pm SEM. 
vs nateglinide $26.5 \pm 5.7 \mathrm{mmol} \cdot \mathrm{hr} / 1, \mathrm{p}<0.003$ ) (Fig. 1 upper panel). Mean total area under the curve of insulin (AUC-IRI) (control $932.6 \pm 356 \mathrm{pmol} \cdot \mathrm{hr} / 1 \mathrm{vs}$ nateglinide $879.0 \pm 339 \mathrm{pmol} \cdot \mathrm{hr} / \mathrm{l}$ ) was almost the same in both groups. But, at $15 \mathrm{~min}$ and $30 \mathrm{~min}$ after glucose load, IRI values were significantly higher, but they were significantly lower on nateglinide than control at $120 \mathrm{~min}, 150 \mathrm{~min}$ and $180 \mathrm{~min}$. AUC-IRI was significantly higher on nateglinide at $0-60 \mathrm{~min}$ (control $175.3 \pm 76.1 \mathrm{pmol} \cdot \mathrm{hr} / \mathrm{l}$ vs nateglinide 332.0 $\pm 90.6 \mathrm{pmol} \cdot \mathrm{hr} / \mathrm{l}, \mathrm{p}<0.001)$ and AUC-IRI was significantly lower on nateglinide at $120-180 \mathrm{~min}$ (control $396.8 \pm 205 \mathrm{pmol} \cdot \mathrm{hr} / 1$ vs nateglinide $226.1 \pm 131 \mathrm{pmol}$. $\mathrm{hr} / \mathrm{l}, \mathrm{p}<0.05)$ (Fig. 1 lower panel).

\section{Discussion}

In the present study the restoration of the impaired early insulin response was established for the first time with nateglinide in obese subjects with type 2 diabetes. The subjects showed delayed hyperinsulinemia with hyperglycemia, demonstrating insulin resistance. Recently it was reported that HOMA-R values are highly correlated with the rates of muscle glucose disposal during clamp study [5]. The average HOMA-R value was 3.33 in the present study indicating relative insulin resistance. However, the glycemic responses of patients during oral glucose load with nateglinide were almost normalized. The pattern of insulin secretion was altered but the gross amount of insulin secretion was not increased.

Bruce et al. reported [3] that restoring early insulin response to the ingestion of meal in type 2 diabetes patients by infusing insulin intravenously reduced postprandial hyperglycemia. Since they infused insulin peripherally, it is likely that muscle and fat cell glucose uptake was enhanced. In the present study, it is speculated that rapidly increased portal insulin concentration suppressed hepatic glucose production, and at the same time more importantly hepatic glucose uptake increased significantly, thus glycemic response after glucose load was normalized with nateglinide in obese type 2 diabetic patients. This would suggest that even though in these patients insulin resistance might exist in muscle, the liver itself might not be insulin resistant. Our results may indicate the importance of portal insulin dynamics in hepatic glucose handling.

\section{Conclusion}

The impairment of early insulin response after glucose load, rather than insulin resistance in peripheral tissues, may be responsible for postprandial hyperglycemia in obese type 2 diabetes.

\section{References}

1. Lillioja S, Mott DM, Howard BV, Bennett PH, YkiJärvinen H, Freymond D, Nyomba BL, Zurlo F, Swinburn B, Bogardus C (1988) Impaired glucose tolerance as a disorder of insulin action: Longitudinal and cross- sectional studies in Pima Indians. $N$ Engl $J$ Med 318: 1217-1225.

2. Reaven G, Miller R (1968) Study of the relationship between glucose and insulin responses to an oral glucose load in man. Diabetes 17: 560-569.

3. Bruce DG, Chisholm DJ, Storlien LH, Kraegen EW (1988) Physiological importance of deficiency in early prandial insulin secretion in non-insulin-dependent diabetes. Diabetes 37: 736-744.

4. Ikenoue T, Okazaki K, Fujitani S, et al. (1997) Effect of a new hypoglycemic agent, A-4166 $\pm(-)$-N-(trans4-isopropylcyclohexanecarbonyl)-D-phenylalanine, on postprandial blood glucose excursion: comparison with voglibose and glibenclamide. Biol Pharm Bull 20: 354-359.

5. Bonora E, Targher G, Alberiche M, Bonadonna RC, Saggiani F, Zenere MB, Monauni T, Muggeo M (2000) Homeostasis model assessment closely mirrors the glucose clamp technique in the assessment of insulin sensitivity. Diabetes Care 23: 57-63. 